\title{
Systematic Pharmacology-Based Strategy to Explore the Molecular Network Mechanism of Modified Taohong Siwu Decoction in the Treatment of Premature Ovarian Failure
}

\author{
Xiao Yuan, ${ }^{1}$ Wang Xiang, ${ }^{1}$ Kailin Yang $\mathbb{D},{ }^{1}$ Huiping Liu $\mathbb{D}^{1},{ }^{1}$ Guomin Zhang $\mathbb{D},{ }^{1}$ Qi He, \\ and Jiapeng Fan ${ }^{2}$ \\ ${ }^{1}$ Hunan University of Chinese Medicine, Changsha, Hunan, China \\ ${ }^{2}$ Harbin Institute of Petroleum, Harbin, Heilongjiang Province, China \\ Correspondence should be addressed to Huiping Liu; 1074983953@qq.com and Guomin Zhang; 834095773@qq.com
}

Received 3 October 2021; Accepted 13 December 2021; Published 21 January 2022

Academic Editor: Yong Wang

Copyright (c) 2022 Xiao Yuan et al. This is an open access article distributed under the Creative Commons Attribution License, which permits unrestricted use, distribution, and reproduction in any medium, provided the original work is properly cited.

\begin{abstract}
Objective. To explore the molecular network mechanism of modified Taohong Siwu Decoction (MTHSWD) to interfere with premature ovarian failure based on systematic pharmacological strategy. Methods. The network pharmacology strategy was used to explore the potential mechanism of MTHSWD intervention in POF, and then it was verified through animal experiments. Mouse zona pellucida 3 was used as an antigen to subcutaneously immunize BALB/c female mice to establish an immune POF model. Mice were divided into MTHSWD low-, medium-, and high-dose groups, positive control group, model group, and normal group. After 30 days of drug intervention, ovarian tissue was taken for pathological hematoxylin-eosin (HE) staining, and immunohistochemical methods were used to detect the expression of TGF- $\beta 1$ and TGF- $\beta$ RII and Smad $2 / 3$ protein expression in follicular wall granular cells and ovarian tissue, respectively. Results. Network pharmacology studies have shown that MTHSWD may interfere with the TGF- $\beta$ signaling pathway. Animal experimental research shows that, compared with the model group, the number of ovarian mature follicles in the MTHSWD groups and the positive group was significantly increased, and the number of atresia follicles decreased. Immunohistochemistry showed that, compared with the control group, the expression of TGF- $\beta 1$, TGF- $\beta$ RII, and Smad2/3 in the follicular wall granulosa cells and ovarian tissues of MTHSWD groups was significantly higher than that of the model group $(P<0.05)$. Conclusion. MTHSWD may improve the ovarian function of POF mice by upregulating the protein expression of granulosa cells TGF- $\beta 1$, TGF- $\beta$ RII, and Smad2/3.
\end{abstract}

\section{Introduction}

Premature ovarian failure (POF) refers to a disease that causes amenorrhea, infertility, menopause, and genitourinary symptoms before the age of 40 due to ovarian failure [1]. Epidemiological studies have shown that the incidence rate in women is about $1 \%[1,2]$. Hormone detection indicators showed that it has hypogonadotropic hypogonadism [3]. At present, hormone replacement therapy (HRT) is the most popular choice for women with POF to get rid of menopausal syndrome [4]. However, HRT has its own indications and contraindications [5]. For example, unexplained vaginal bleeding, acute liver injury, liver insufficiency, vascular embolism, and breast cancer are contraindications to HRT $[6,7]$. In alternative medicine, ancient Chinese medicine has accumulated a lot of clinical experience. With the increase of clinical evidence, TCM has shown a good effect in the treatment of POF [8-10]. The systematic review and meta-analysis of Bushen Huoxue (nourishing the kidney and promoting blood circulation) Chinese herbal medicine for POF showed that compared with the western medicine group, Chinese medicine may improve the total effective rate, menstrual improvement rate, symptom score improvement, and so on, and the incidence of adverse 
reactions is low $[11,12]$. Nourishing the kidney and promoting blood circulation, Chinese medicine may also reduce the level of follicle-stimulating hormone, increase the level of estrogen, reduce clinical symptoms, promote the growth of antral follicles, increase the volume of the ovary, increase the blood flow speed and blood flow pulsation of the ovary, and reduce the blood flow resistance of the ovary. It may also reduce the level of osteocalcin and serum alkaline phosphatase in the body, increase the level of calcitonin, delay the occurrence of osteoporosis, reduce triglycerides and total cholesterol, and reduce the risk of cardiovascular and cerebrovascular diseases. It may also increase the level of CD3+ and CD4+ lymphocytes, improve the body's immunity, and reduce the recurrence rate after drug withdrawal [13-16].

Siwu Decoction [17] was first published in the "Secret Recipe of Xianshou Li Shang" by Lin Taoist in the Tang Dynasty. It was used to treat traumatic diseases, iron beating injuries, blood loss, and blood stasis. In the Song Dynasty, “Tai Ping Hui Min He Ji Ju Fang" began to develop Siwu Decoction into a special prescription for the treatment of gynecological diseases, enriching blood, promoting blood circulation, and regulating menstruation $[18,19]$. Since then, physicians of the past generations have elaborated and exerted the use of Siwu Decoction in the treatment of gynecological diseases. They believed that the effect of Siwu Decoction was to enrich blood, promote blood circulation, regulate menstruation, and treat many diseases caused by blood deficiency and blood addiction [19]. On this basis, the addition and subtraction changes have formed many Siwu Decoctions as the core to treat women's abdominal pain during menstruation, that is, Siwu Decoction prescriptions for gynecological blood stasis dysmenorrhea [20]. Among them, Taohong Siwu Decoction is the main representative prescription of nourishing blood and promoting blood circulation in Siwu Decoctions. Recent studies have shown that Modified Taohong Siwu Decoction (MTHSWD) combined with HRT may significantly improve ovarian function and improve the clinical efficacy of the treatment of POF [21]. MTHSWD can improve the symptoms of late menstruation, decreased menstrual flow, irritability, and vaginal dryness in patients with decreased ovarian reserve, reduce FSH levels, improve ovarian blood supply, and increase the number of antral follicles [22]. In addition, the treatment of infertility patients with Shou Tai Wan combined with Taohong Siwu Decoction can effectively improve the quality of pregnancy and improve the immune function and ovarian function of the body [23]. Further studies have shown that Siwu Decoction can improve ovarian reserve and improve follicular dysplasia [24, 25].

However, the molecular biological network mechanism of MTHSWD in the treatment of POF is still not clear. Network pharmacology combines the ideas of systems biology and multidirectional pharmacology. It analyzes the mechanism of action of the effective ingredients of drugs by constructing a complex network within "component targetspathways-disease," which turns pharmacological research from the traditional research concept of finding a single target to the network comprehensive analysis thinking $[26,27]$. In network pharmacology research, the same disease can be regulated by different genes at different stages of development, and some genes can also play a central regulatory role in multiple diseases. This coincides with the traditional Chinese medicine theory of "different treatment of the same disease" and "treatment of the same disease at the same time" [28, 29]. Therefore, this study hopes to explore the molecular biological network effect and the basis of pharmacodynamic active ingredients of MTHSWD in the treatment of POF by combining systemic pharmacology and experimental pharmacology strategies, so as to provide a scientific basis for the clinical application of MTHSWD.

\section{Materials and Methods}

2.1. Potential Compounds and Targets of MTHSWD and POF Gene Collection. The potential components and targets of MTHSWD were searched from TCMSP (https://tcmsp-e. $\mathrm{com} /$ ) [30] according to the pharmacokinetic parameters of chemical components (absorption, distribution, metabolism, and excretion (ADME)). The standard was oral bioavailability $(\mathrm{OB}) \geq 30 \%$, Caco- 2 parameter $>-0.4$, and drug-like activity $(\mathrm{DL}) \geq 0.18$ [30]. The POF genes were collected to search the Online Mendelian Inheritance in Man (OMIM, http://omim.org/) [31] and GeneCards (http:// www.genecards.org/) [32]. The official gene symbol of MTHSWD potential targets and POF genes were collected from UniProt KB (https://www.uniprot.org/uniprot/), with the species restricted to human (Table S1 and Table S2).

2.2. Network Construction and Analysis Methods. The String database (https://string-db.org/) was utilized to collect the PPI data of MTHSWD targets and POF genes [33]. The Cytoscape 3.7.2 was utilized to construct and analyze the MTHSWD-POF PPI network [34]. The DAVID ver 6.8 (https://david.ncifcrf.gov/) was utilized to perform gene ontology (GO) enrichment and Kyoto Encyclopedia of Genes and Genomes (KEGG) pathway analysis [35].

\subsection{Experimental Materials}

2.3.1. Experimental Animal. Sixty-seven healthy female BALB/c mice (SYXK (Xiang) 2013-0005), aged 7-8 weeks and weighing 20-22 g, were selected. The vaginal exfoliated cell smear showed that the estrus cycle was normal. Mice were kept in a clean environment, room temperature $18 \sim 22^{\circ} \mathrm{C}$, relative humidity $40 \% \sim 60 \%$, and light for $12 \mathrm{~h}$.

2.3.2. Experimental Drugs. MTHSWD is composed of Rehmanniae Radix Praeparata (Di Huang) 15 g, Polygonatum sibiricum Red. (Huang Jing) $12 \mathrm{~g}$, Cornus officinalis Sieb. et Zucc. (Shan Zhu Yu) 12 g, Lycium barbarum L. (Gou Qi Zi) 12 g, Angelica sinensis (Oliv.) Diels (Dang Gui) 15 g, Paeonia lactiflora Pall. (Bai Shao) $12 \mathrm{~g}$, Ligusticum chuanxiong Hort. (Chuan Xiong) 9 g, Salvia miltiorrhiza Bge. (Dan Shen) 12 g, and Prunus persica (L.). Batsch (Tao Ren) $9 \mathrm{~g}$. Those medical materials were purchased from Pharmacy Department of the First Affiliated Hospital of Hunan University of Chinese Medicine. The Department of Pharmacy, the First Affiliated 
Hospital of Hunan University of Chinese Medicine, identified, screened, washed, processed, sliced, dried, and crushed the source and variety of the same batch of Chinese medicinal materials. The batch number was 20150701. Estradiol valerate tablets were obtained from Bayer Healthcare Co., Ltd., National Medicine Standard J20130009.

2.3.3. Instruments and Reagents. Mouse zona pellucida polypeptide solution and the 330-342 amino acid sequence of mouse zona pellucida 3 (ZP3) (NSSSSQFQIHGPR), with an analytical purity of $91.5 \%$, were obtained from Hangzhou Zhongpi Biochemical Co., Ltd. (P00105). Freund's complete adjuvant (F5881) and Freund's incomplete adjuvant (F5506) were obtained from American Sigma company. Rabbit antimouse TGF- $\beta 1$, TGF- $\beta$ RII, and Smad $2 / 3$ polyclonal antibodies were obtained from Wuhan Boster Bioengineering Co., Ltd. (BA0290, BA0526, BA1395). TRIzol reagent was obtained from Thermo Fisher Technology (China) Co., Ltd. [lot number 267309]. MonScri RTIII All-in-One Mix with sdDNase was obtained from Mona Biotechnology Co., Ltd. (lot number 130449). Universal SYBR qPCR Master Mix Universal Real-Time PCR Kit was obtained from China Biosharp Company (lot number 70090100). Horseradish peroxidase- (HRP-) labeled secondary antibody was obtained from Beijing Zhongshan Jinqiao Biotechnology Co., Ltd. (ZDR-5118). Whole protein extraction kit was obtained from Nanjing KGI Biotechnology Development Co., Ltd. (KGP2100). BCA protein quantitative detection kit was obtained from Shanghai Shenggong Biological Engineering Co., Ltd. (C503031). LEICADMLB2 binocular microscope and LEICARM2255 automatic rotary microtome were obtained from German LEICA company.

\subsection{Quality Control of MTHSWD}

2.4.1. Preparation of Sample. Control solution preparation: morroniside $4.07 \mathrm{mg}$, loganin $1.16 \mathrm{mg}$, and paeoniflorin $2.34 \mathrm{mg}$ were accurately weighed into a $10 \mathrm{~mL}$ volumetric flask. Then methanol : water $(1: 1)$ was added, dissolved, and diluted to the mark and filtered through a $0.45 \mu \mathrm{m}$ filter membrane to make a mixed reference solution.

MTHSWD solution preparation: the medicinal materials of MTHSWD were placed in a round-bottomed flask, $1000 \mathrm{~mL}$ of pure water was added, and the mixture was refluxed, extracted for $1.5 \mathrm{~h}$, and filtered. Then, $1000 \mathrm{~mL}$ of pure water was added, refluxed for extraction for $1 \mathrm{~h}$, and filtered. The two filtrates were combined and concentrated to $500 \mathrm{~mL}$ by rotary evaporation. $2 \mathrm{~mL}$ of the concentrated filtrate was added into a $5 \mathrm{~mL}$ volumetric flask, and anhydrous methanol was added to the volume. Then, the filtrate was ultrasonically processed (power $300 \mathrm{~W}$, frequency $40 \mathrm{~Hz}$ ) for $30 \mathrm{~min}$ and filtered by suction. The subsequent filtrate was filtered with a $0.45 \mu \mathrm{m}$ organic microporous filter membrane.

2.4.2. HPLC Condition. Chromatographic column was Hypersil ODS C18 chromatographic column $(200 \mathrm{~mm}$ $\times 4.6 \mathrm{~mm}, 5 \mu \mathrm{m})$. Flow rate was $1.0 \mathrm{~mL} / \mathrm{min}$. Detection wavelength was $237 \mathrm{~nm}$. Mobile phase was acetonitrile (A)$0.1 \%$ phosphoric acid solution (B) gradient elution. Injection volum was $10 \mu \mathrm{L}$. Column temperature was $30^{\circ} \mathrm{C}$. The contents of those components are morroniside $4.612 \pm 0.013 \mathrm{mg} / \mathrm{g}$, loganin $1.291 \pm 0.003 \mathrm{mg} / \mathrm{g}$, and paeoniflorin $3.084 \pm 0.009 \mathrm{mg} /$ g (Figure S2).

\subsection{Experimental Methods}

2.5.1. Experimental Grouping, Modeling, and Intervention. According to the random number table method, 67 female $\mathrm{BALB} / \mathrm{c}$ mice were divided into (N) 10 mice as the blank group, and the remaining 57 were prepared for modeling. Add $6 \mathrm{mg}$ of ZP3 transparent polypeptide powder, add $6 \mathrm{~mL}$ of doubledistilled water to make a solution, and make a $1: 1$ immune reagent with Freund's complete adjuvant; it is formulated with Freund's incomplete adjuvant at a ratio of $1: 1$ to prepare immune enhancement reagents (both in the form of porous white viscous oil). Mice were given $0.15 \mathrm{~mL}$ of immune reagent injected into the soles of both hind feet and subcutaneously in the abdominal cavity. 14 days later, $0.15 \mathrm{~mL}$ of immunoenhancing reagent was injected again into the soles of both hind feet and subcutaneously in the abdominal cavity to establish an immune POF model. Mice in the blank group was injected with $0.15 \mathrm{~mL}$ of normal saline into the same area. Beginning on the 8th day after modeling, all mice were subjected to cervical mucus smears and HE staining of vaginal exfoliated cells at 9:00 every morning. The observation lasted for 12 days and the estrus cycle of the mice was checked. The results showed that 5 mice still did not have any estrous cycle disorder and were eliminated.

In the remaining 52 mice, 2 were randomly selected to observe follicular morphology to confirm the POF model. The other 50 mice were randomly divided into model group, positive control group, MTHSWD low-dose group, middledose group, and high-dose group, with 10 mice in each group. The dosages of MTHSWD low-, medium-, and highdose groups were $0.54 \mathrm{~g}, 1.08 \mathrm{~g}$, and $2.16 \mathrm{~g}$ of crude $\mathrm{drug} / \mathrm{mL}$, respectively. The low, medium, and high doses of MTHSWD were converted by 1,2 , and 4 times the adult clinical dose according to the "Equivalent Dose Table for Conversion of Human and Animal Body Surface Areas," respectively $[36,37]$. The positive control group was given $0.03 \mathrm{mg}$ of Estradiol. The intragastric administration was started 1 week after the model was established. Both the blank group and the model group were given $0.3 \mathrm{~mL}$ of normal saline. The intervention lasted 30 days and was given by gavage. The mice were weighed every 7 days.

2.5.2. Pathological Observation. The ovaries were fixed in $4 \%$ paraformaldehyde solution, dehydrated with gradient alcohol, embedded in paraffin, sectioned, deparaffinized, and stained with the conventional hematoxylin-eosin (HE) method.

2.5.3. Expression of TGF- $\beta 1$, TGF-B $\beta R I I$, and Smad $2 / 3$
Protein in Ovarian Tissue Detected by
Immunohistochemistry. The ovaries were fixed in $4 \%$


paraformaldehyde solution, dehydrated with gradient alcohol, embedded in paraffin, sectioned, and deparaffinized. Then the expression of TGF- $\beta 1$, TGF- $\beta$ RII, and Smad $2 / 3$ protein in ovarian tissue was detected by immunohistochemistry SP two-step method. Then the areas under the microscope were randomly selected and analyzed with Image-Pro Plus 6.0. The cumulative optical density (IOD) and area and mean density were measured according to the standard operation method.

2.5.4. Determination of Smad2, Smad3, and Smad7 mRNA Expression in Ovarian Tissue. The total RNA of mouse ovarian tissue was extracted according to the TRIzol method, and the first-strand cDNA was synthesized by reverse transcription, and Smad2, Smad3, and Smad7 mRNA were detected according to the Real-Time PCR method, and $\beta$-actin was used as the internal control. The reaction was prepared according to the operating instructions of the kit, and the primer sequence was synthesized by Shenggong Bioengineering (Shanghai) Co., Ltd. The reaction conditions were $95^{\circ} \mathrm{C}$ predenaturation for $2 \mathrm{~min}, 95^{\circ} \mathrm{C}$ denaturation for $15 \mathrm{~s}, 60^{\circ} \mathrm{C}$ annealing for $20 \mathrm{~s}, 72^{\circ} \mathrm{C}$ extension for $30 \mathrm{~s}$, and 40 cycles of amplification. The 2- $\triangle \triangle \mathrm{Ct}$ method was used to analyze mRNA expression levels (Table 1 ).

2.6. Statistical Analysis. The SPSS 21.0 statistical software was used for analysis, and the data were expressed as mean \pm standard deviation. Multigroup analysis was performed by single-factor analysis of variance. $P<0.05$ indicated that the difference was statistically significant.

\section{Results}

3.1. MTHSWD Potential Targets and POF Targets. A total of 247 MTHSWD potential targets were obtained and 754 POF genes were collected from OMIM and GeneCards. The relationship among compounds and targets of MTHSWD is shown in Figure 1. This network consists of 100 compound nodes, 247 potential target nodes, and 1527 edges. In this network, the targets near the center can be regulated by more components than targets near the periphery.

3.2. MTHSWD-POF PPI Network Analysis. The MTHSWD potential targets, POF genes, and the PPI data were input into Cytoscape 3.7.2 to construct MTHSWD-POF PPI network. This network consists of 823 nodes (578 POF gene nodes, 183 MTHSWD potential target nodes, and 62 MTHSWD-POF target nodes) and 19442 edges. The targets are arranged according to degree from large to small, and the top 20 targets can be divided into 3 categories: (1) MTHSWD potential targets: JUN, EGFR, IL1B, EGF, HIF1A, and FOS; (2) POF genes: ACTB, ALB, INS, and IGF1; (3) MTHSWDPOF targets: TP53, AKT1, IL6, MYC, TNF, ESR1, VEGFA, STAT3, CASP3, and PTEN (Figure 2).

\subsection{Enrichment Analysis of MTHSWD-POF PPI Network}

3.3.1. Biological Processes of MTHSWD-POF PPI Network. The biological processes include positive regulation of pathway-restricted SMAD protein phosphorylation, positive regulation of transcription from RNA polymerase II promoter, response to drug, SMAD protein signal transduction, positive regulation of DNA-templated transcription, aging, response to ethanol, positive regulation of gene expression, response to hypoxia, positive regulation of cell proliferation, cholinergic synaptic transmission BMP signaling pathway, negative regulation of apoptotic process, ovarian follicle development, regulation of apoptotic process, transforming growth factor beta receptor signaling pathway, and signal transduction (Figure 3) (Table S3).

\subsubsection{Cell Components of MTHSWD-POF PPI Network.} The cell components include extracellular space, cytosol, extracellular region, acetylcholine-gated channel complex, receptor complex, nucleoplasm, integral component of plasma membrane, postsynaptic membrane, membrane raft, external side of plasma membrane, transcription factor complex, cell surface, plasma membrane, neuron projection, and cytoplasm (Figure 4) (Table S3).

\subsubsection{Molecular Function of MTHSWD-POF PPI Network.} The molecular function includes transforming growth factor beta receptor binding, growth factor activity, enzyme binding, cytokine activity, protein binding, protein homodimerization activity, drug binding, acetylcholine binding, acetylcholine-activated cation-selective channel activity, hormone activity, identical protein binding, acetylcholine receptor activity, ligand-gated ion channel activity, and protein heterodimerization activity (Figure 5) (Table S3).

\subsubsection{Signaling Pathway of MTHSWD-POF PPI Network.} The signaling pathway includes neuroactive ligand-receptor interaction, TGF-beta signaling pathway, FoxO signaling pathway, ovarian steroidogenesis, TNF signaling pathway, prolactin signaling pathway, apoptosis, PI3K-Akt signaling pathway, T cell receptor signaling pathway, steroid hormone biosynthesis, HIF-1 signaling pathway, cytokine-cytokine receptor interaction, neurotrophin signaling pathway, p53 signaling pathway, NF-kappa B signaling pathway, and NOD-like receptor signaling pathway (Figures 6 and 7). The TGF-beta signaling pathway was shown in Figure 8 (Table S3).

3.4. Morphological Changes of Ovarian Tissue. In model group, the ovarian volume was reduced; a few primary follicles and growing follicles were seen in the ovarian cortex. The number of mature follicles was significantly reduced, the atretic follicles increased, and the secondary follicles were loosely arranged. In MTHSWD mediumand low-dose groups, compared with the model group, 
TABLE 1: Primer sequence of RCR.

\begin{tabular}{lrr}
\hline Sequence & Upstream $5^{\prime}-3^{\prime}$ & ${\text { Downstream } 5^{\prime}-3^{\prime}}$ \\
\hline TGF- $\beta 1$ & CCAAGGAGACGGAATACAGG & GTGTTGGTTGTAGAGGGCAAG \\
Smad 2 & AGCCGCCCGAAGGGTA & AGACCCACCGGCTGATTTTT \\
Smad 3 & CGAGCTGCCTCTGTGCG & CCATCCAGTGACCTGGGGAT \\
$\beta$-Asctin & CGCGAGTACAACCTTCTTGC & CGTCATCCATGGCGAACTGG \\
\hline
\end{tabular}

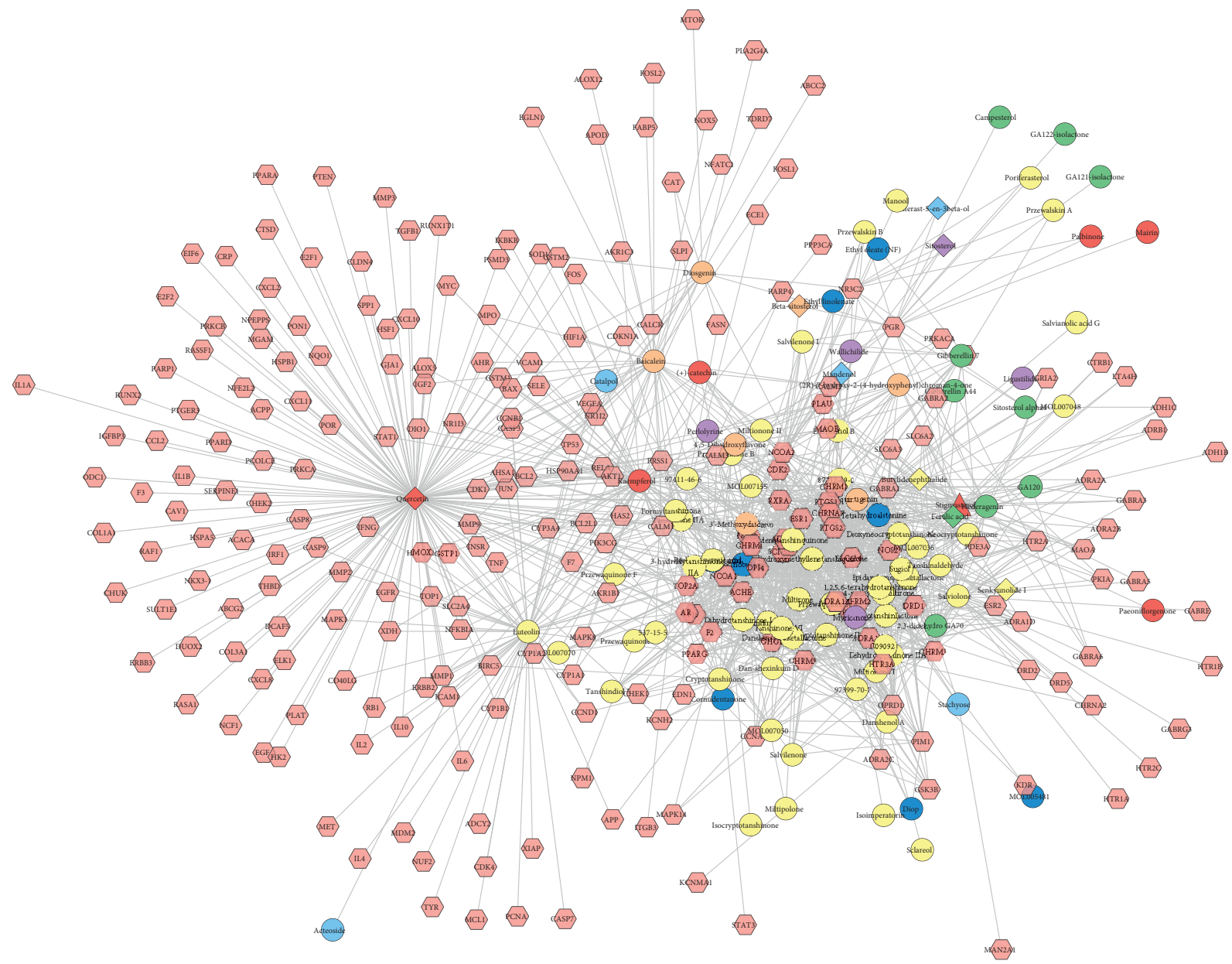

Figure 1: Compound-compound target of MTHSWD (red, orange, yellow, green, blue, indigo, and purple circles stand for components of Paeonia lactiflora Pall., Polygonatum sibiricum Red., Salvia miltiorrhiza Bge., Prunus persica (L.) Batsch, Rehmanniae Radix Praeparata, Cornus officinalis Sieb. et Zucc., and Ligusticum chuanxiong Hort., respectively. Red diamond stands for component of Lycium barbarum L. Orange diamond stands for common component of Polygonatum sibiricum Red., Prunus persica (L.) Batsch, Rehmanniae Radix Praeparata, Cornus officinalis Sieb. et Zucc., and Angelica sinensis (Oliv.) Diels. Yellow diamond stands for the common components of Angelica sinensis (Oliv.) Diels and Ligusticum chuanxiong Hort. Green diamond stands for the common components of Angelica sinensis (Oliv.) Diels and Lycium barbarum L. Blue diamond stands for the common components of Ligusticum chuanxiong Hort. and Cornus officinalis Sieb. et Zucc. Indigo diamond stands for the common components of Cornus officinalis Sieb. et Zucc. and Salvia miltiorrhiza Bge. Purple diamond stands for the common components of Paeonia lactiflora Pall., Polygonatum sibiricum Red., Cornus officinalis Sieb. et Zucc., and Ligusticum chuanxiong Hort. Red triangle stands for the common components of Rehmanniae Radix Praeparata, Cornus officinalis Sieb. et Zucc., and Angelica sinensis (Oliv.) Diels.).

the number of primary and mature follicles increased and the number of atretic follicles decreased. In MTHSWD high-dose group and the positive control group, a large number of primary follicles and antral follicles are seen in the ovarian cortex, and there are many near-mature follicles, and the corpus luteum increases and grows well (Figure 9).
3.5. Expression of TGF- $\beta 1$, Smad2, and Smad3 mRNA in Ovarian Tissue. Compared with the normal group, the expression levels of TGF- $\beta 1$, Smad2, and Smad3 mRNA in the ovarian tissue of the model group were significantly decreased $(P<0.05)$. Compared with the model group, the expression levels of TGF- $\beta 1$, Smad2, and Smad 3 mRNA in the ovarian tissue of the MTSWD group increased $(P<0.05)$ (Figure 10). 


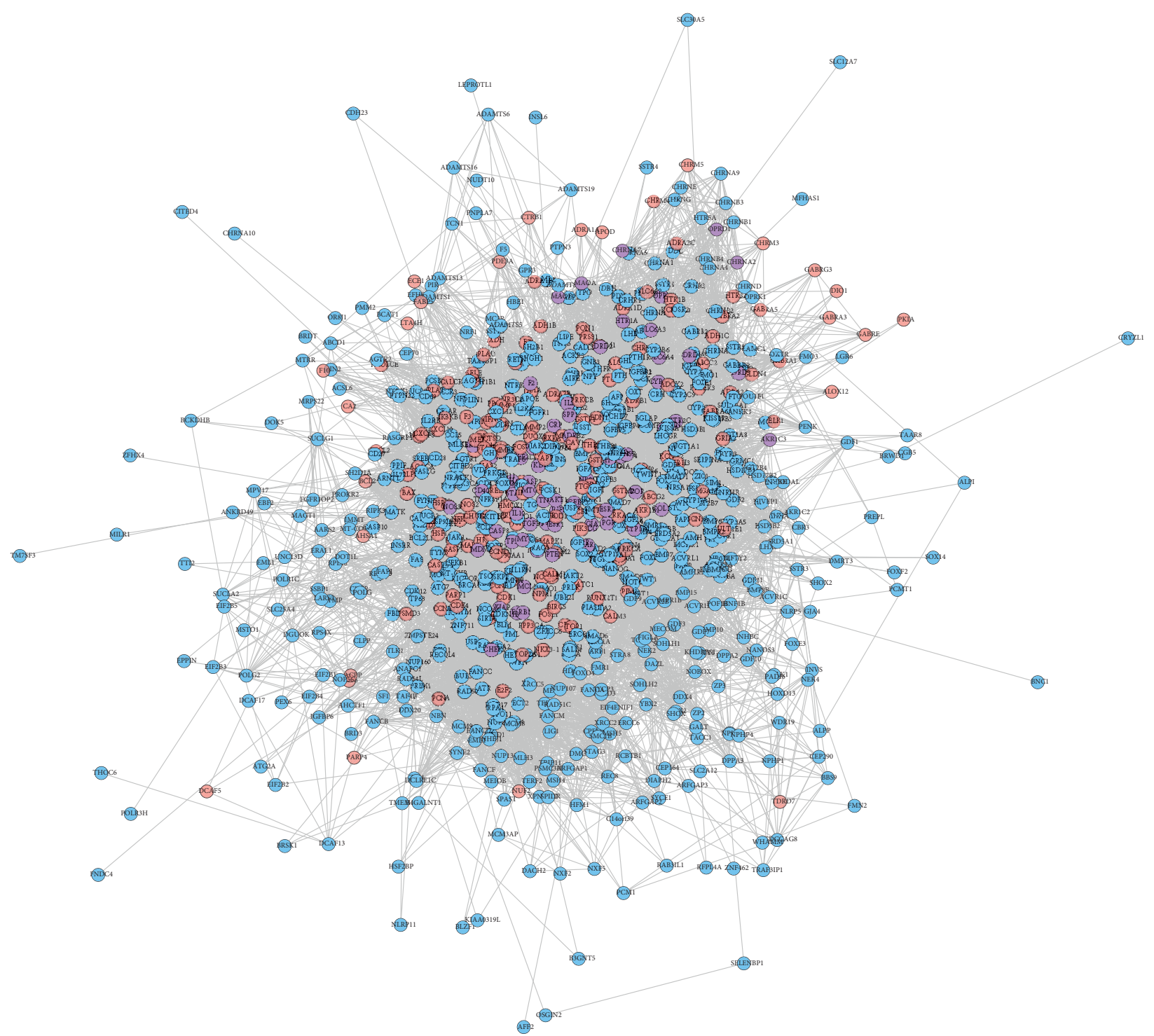

FIGURE 2: MTHSWD-POF PPI network (blue, pink, and purple circles stand for POF gene, MTHSWD target, MTHSWD-POF target, resp.).

3.6. Expression of TGF- $\beta 1, T G F-\mathrm{B} \beta R I I$, and Smad2/3 in Granulosa Cells of Ovarian Follicle Wall Detected by Immunohistochemistry. In the model group, TGF- $\beta 1$, Smad2/3, and TGF- $\beta$ RII were weakly expressed in the cytoplasm of granulosa cells of antral follicles. In the MTHSWD low-dose and middle-dose groups, TGF- $\beta 1$ and Smad2/3 were strongly expressed in some areas of antral follicles. In the MTHSWD high-dose group, positive control group, and blank group, TGF- $\beta 1$ and Smad2/3 were strongly expressed in most areas of antral follicles. TGF- $\beta$ RII was strongly expressed in some areas of the antral follicles of MTHSWD low-, medium-, and high-dose groups, positive control group, and blank group (Figures 11-13).

Compared with the model group, the expression of TGF- $\beta 1$, TGF- $\beta$ RII, and Smad $2 / 3$ in the MTHSWD medium-dose, high-dose group, positive control group, and blank group increased $(P<0.05)$. There was no difference in the expression of TGF- $\beta 1$, TGF- $\beta$ RII, and Smad $2 / 3$ in the MTHSWD medium-dose and high-dose groups compared with the positive control group $(P<0.05)$ (Figure 14).

\section{Discussion}

At present, it is more recognized that the factors that cause POF involve genetics, immunity, infection, iatrogenic, psychological, and other factors. In the treatment of POF, hormone replacement or combined use of ovulation induction and assisted reproductive technology is also used for those who have fertility requirements [38]. Previous studies have shown that TCM, which focuses on invigorating the kidney, can reduce the damage of cisplatin to the ovary, promote follicular development, and improve and enhance ovarian function by regulating the content of various hormones in the serum of POF rat models and changing the expression of apoptotic cell-related proteins [39]. Further studies have shown that the Chinese 


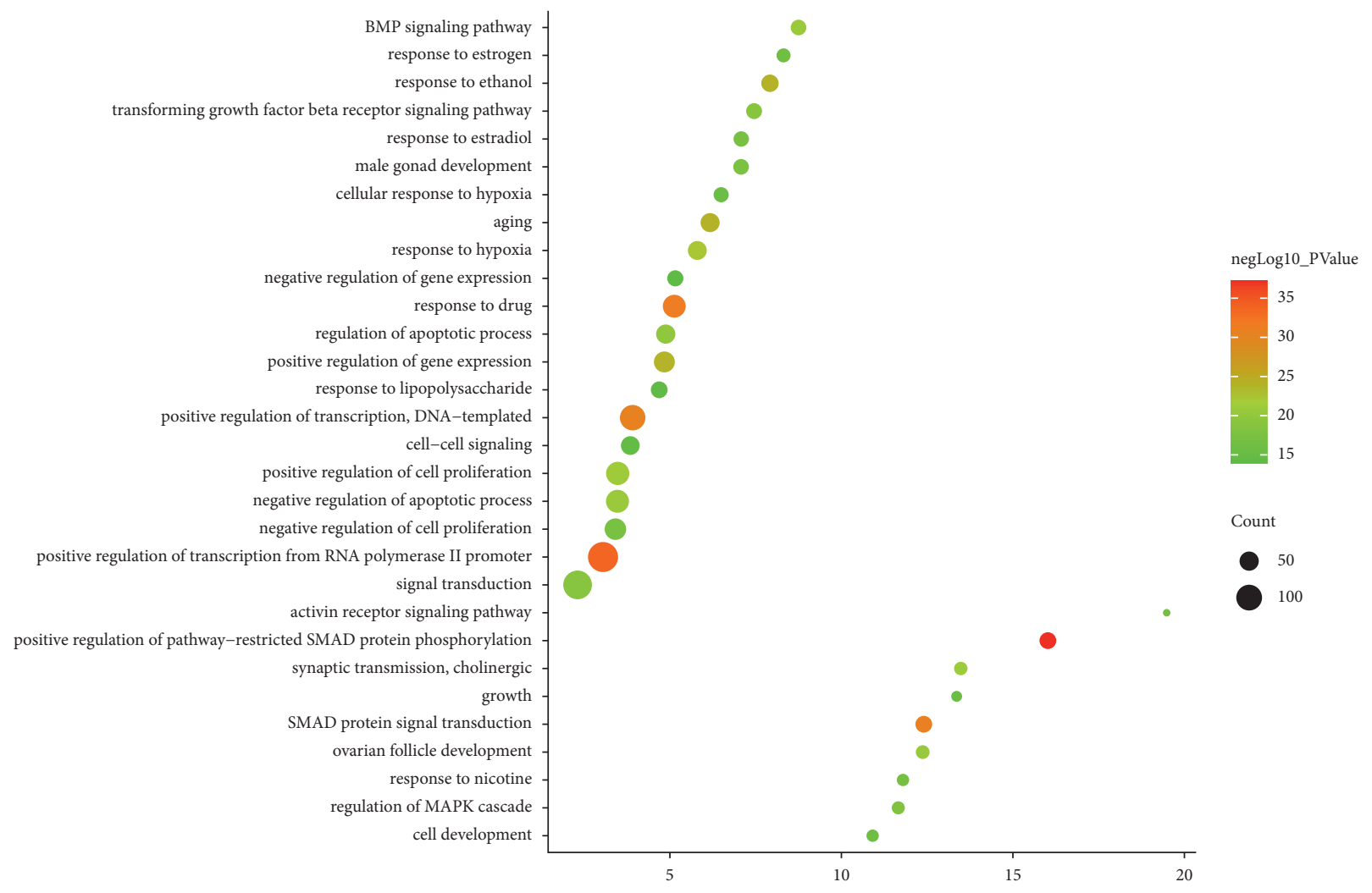

Figure 3: Bubble chart of biological processes ( $X$-axis stands for fold enrichment).

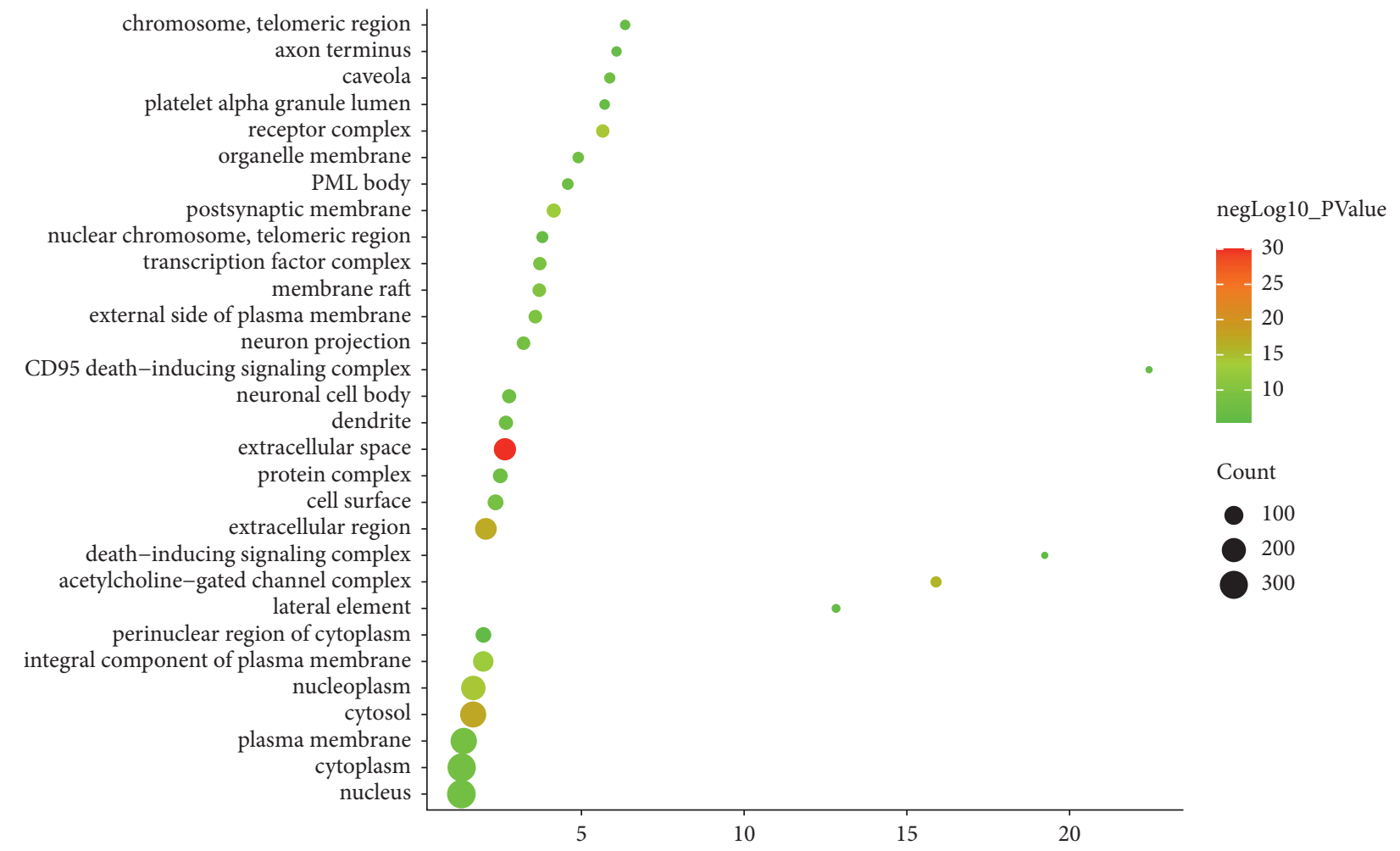

FIgURE 4: Bubble chart of cell components ( $X$-axis stands for fold enrichment). 


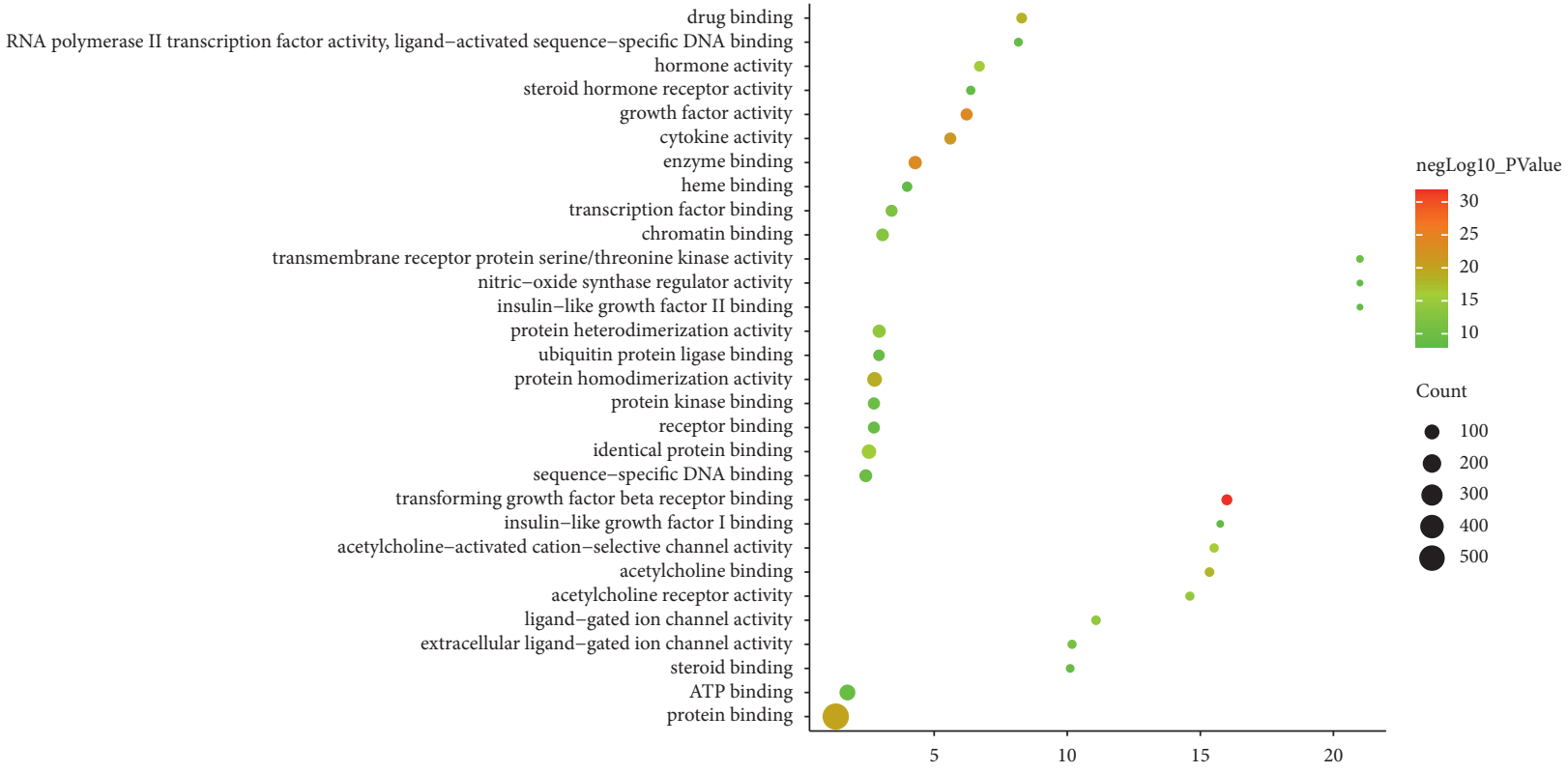

Figure 5: Bubble chart of molecular function ( $X$-axis stands for fold enrichment).

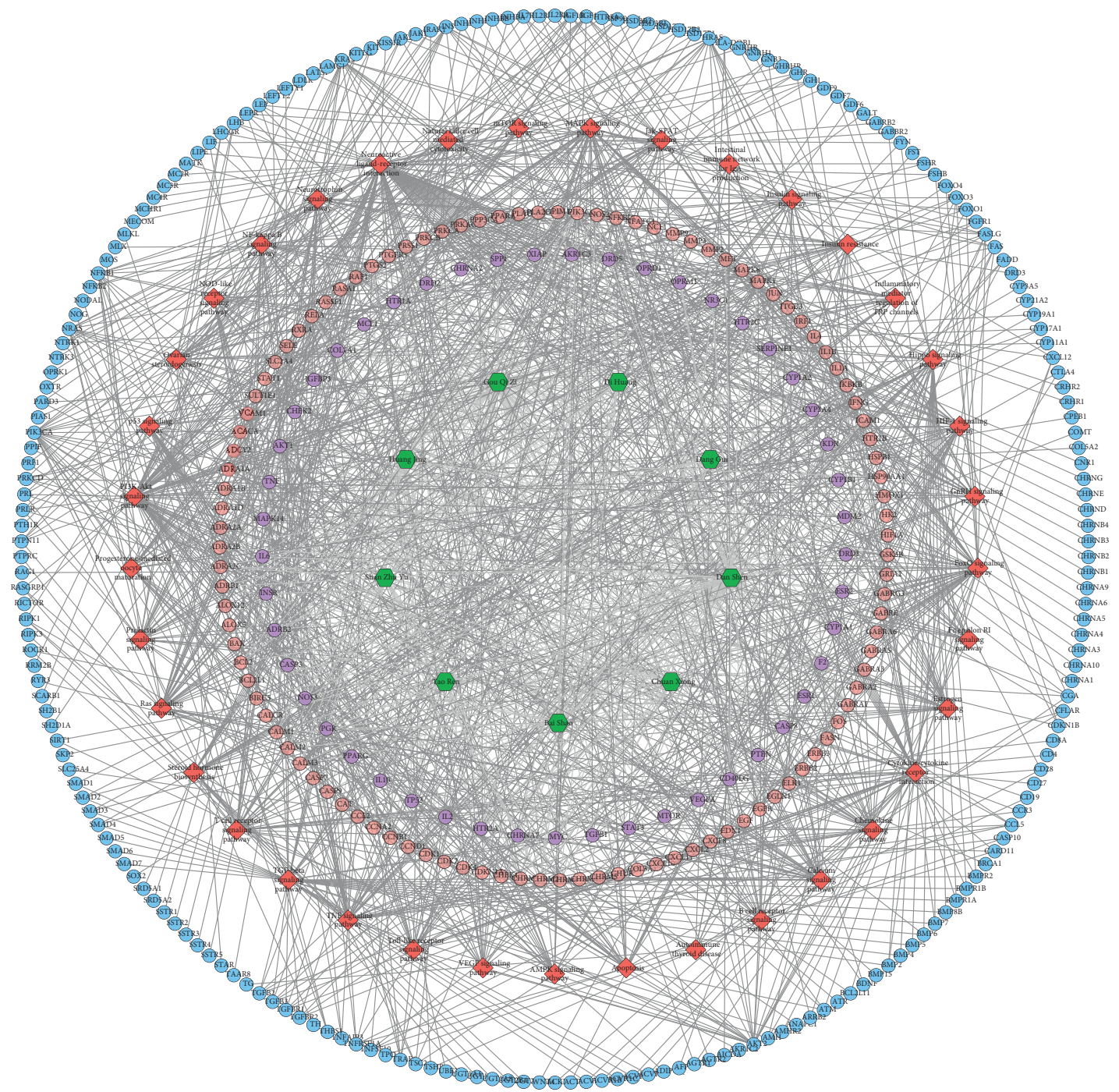

Figure 6: Herb-Target-Signaling pathway network (red diamond stands for signaling pathway. Blue, pink, and purple circles stand for POF gene, MTHSWD target, and MTHSWD-POF target, respectively. Green hexagon stands for herbs). 


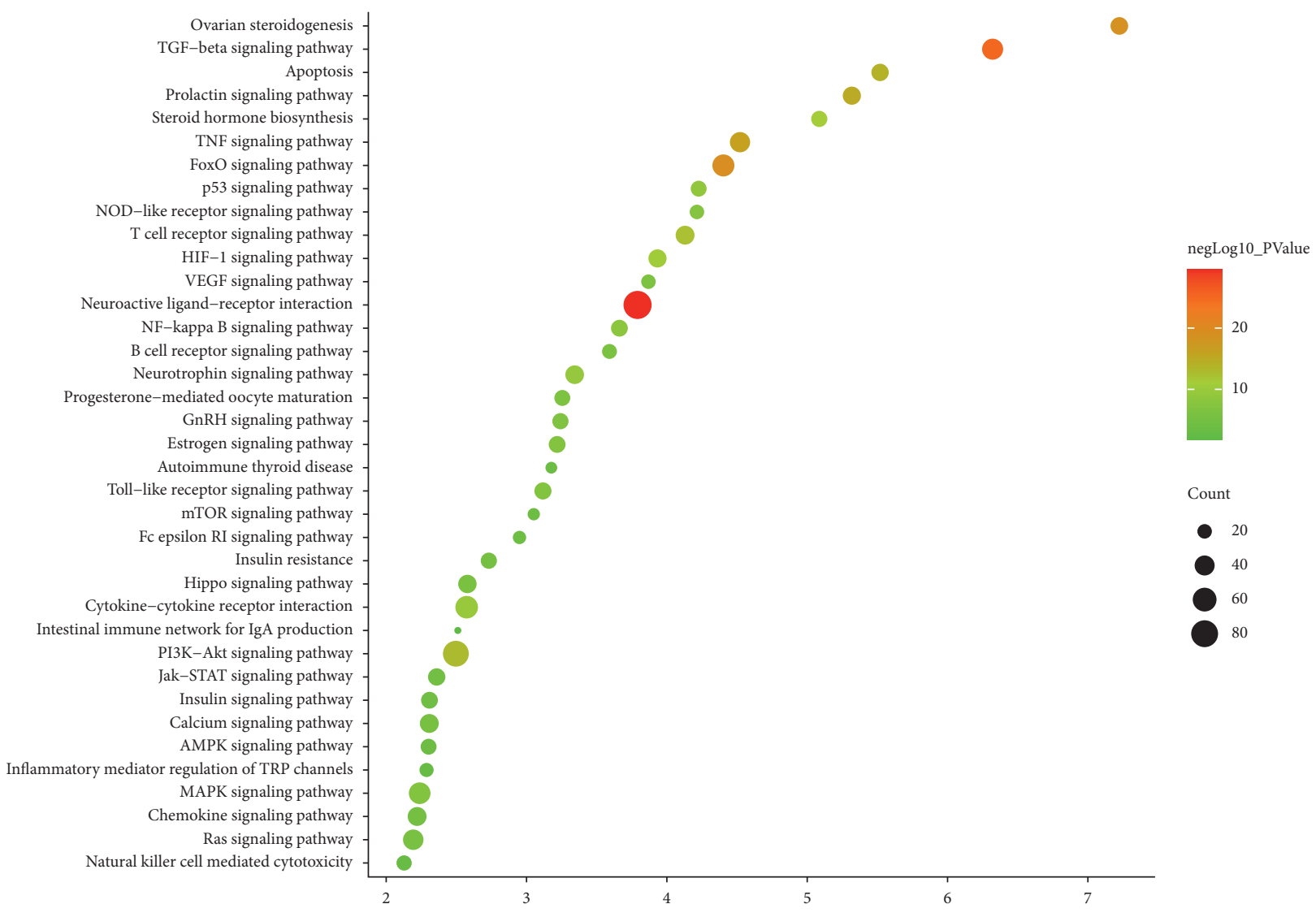

Figure 7: Bubble diagram of signaling pathways ( $X$-axis stands for fold enrichment).

medicine compound for replenishing qi and blood can regulate the rate of estrous cycle disorder in mice with primary ovarian insufficiency, reduce serum $\mathrm{LH}$ and $\mathrm{FSH}$ levels, and reduce the rate of ovarian granulosa cell apoptosis. It may also increase serum E2 and AMH levels, increase the quality of uterus and ovaries, and increase the expression of TGF- $\beta$, GDF-9, and BMP15 proteins in ovarian tissue, thereby improving the ovarian function of POF mice [40].

Current studies have shown that mutations in the bone morphogenetic proteins (BMP) gene, which is a member of the transforming growth factor (TGF- $\beta$ ) superfamily, will cause serious abnormalities in follicular development and ovulation. The TGF- $\beta /$ Smad signaling pathway involves the growth and development of follicles, the proliferation and apoptosis of granulosa cells and membrane cells, the synthesis of steroid hormones, the maturation of oocytes, ovulation, and luteinization [41, 42]. Smad2 and Smad3 proteins are members of the Smads protein family receptor regulation, and their main function is to participate in signal transduction in the TGF- $\beta$ and activin signaling pathways. At present, siRNA transfection studies have shown that Smad2 and Smad3 are involved in the upregulation of TGF- $\beta 1$ and the production of PGE2, respectively, and then participate in the occurrence and development of ovarian regulation of ovulation [41]. Further studies have shown that Smad2/3 plays an important role in the transition from primordial follicles to primary follicles and the formation of antral follicles [43-45]. The fertility of mice with knocked out Smad2 and Smad3 genes was greatly reduced, and they cannot form normal cumulus expansion and mediate the signal transduction between granulosa cells and oocytes [46]. Researches have showed that Bushen Tiaochong method can effectively increase the expression of TGF- $\beta 1, \operatorname{Smad} 3$, and $\mathrm{P}-\mathrm{Smad} 3$ in rat ovarian cells [46]. However, the specific role and mechanism of TGF- $\beta /$ Smad signaling pathway in the granulosa cells of immune POF mice have not been reported yet. The factors that affect follicle development and cell proliferation and differentiation involve multiple signaling pathways, including wnt $\beta$-catenin signaling pathway [47], Nodal signaling pathway [48], FGF signaling pathway [49], and BMP/Smads signaling pathway [50]. Smad2 and Smad3 are important factors for maintaining ovarian development and function $[51,52]$. A number of studies have shown that the fertility of Smad3 knockout mice is reduced, and the proliferation of granulosa cells is inhibited $[53,54]$. Smad2/3 plays an important factor in the granular cells of newly formed primordial follicles [55]. Smad 2 and Smad3 knockout mice have greatly reduced fertility [45]. Yang et al. further found that TGF- $\beta$ inhibits the degradation of CyclinD2 through Smad2 and Smad3, activates CDK4, and promotes the synthesis of granular cell DNA [56]. This study found that, compared with the blank group, the protein expression of granulosa cells TGF- $\beta 1$, TGF- $\beta$ RII, and Smad $2 / 3$ in the mouse follicles of the model 


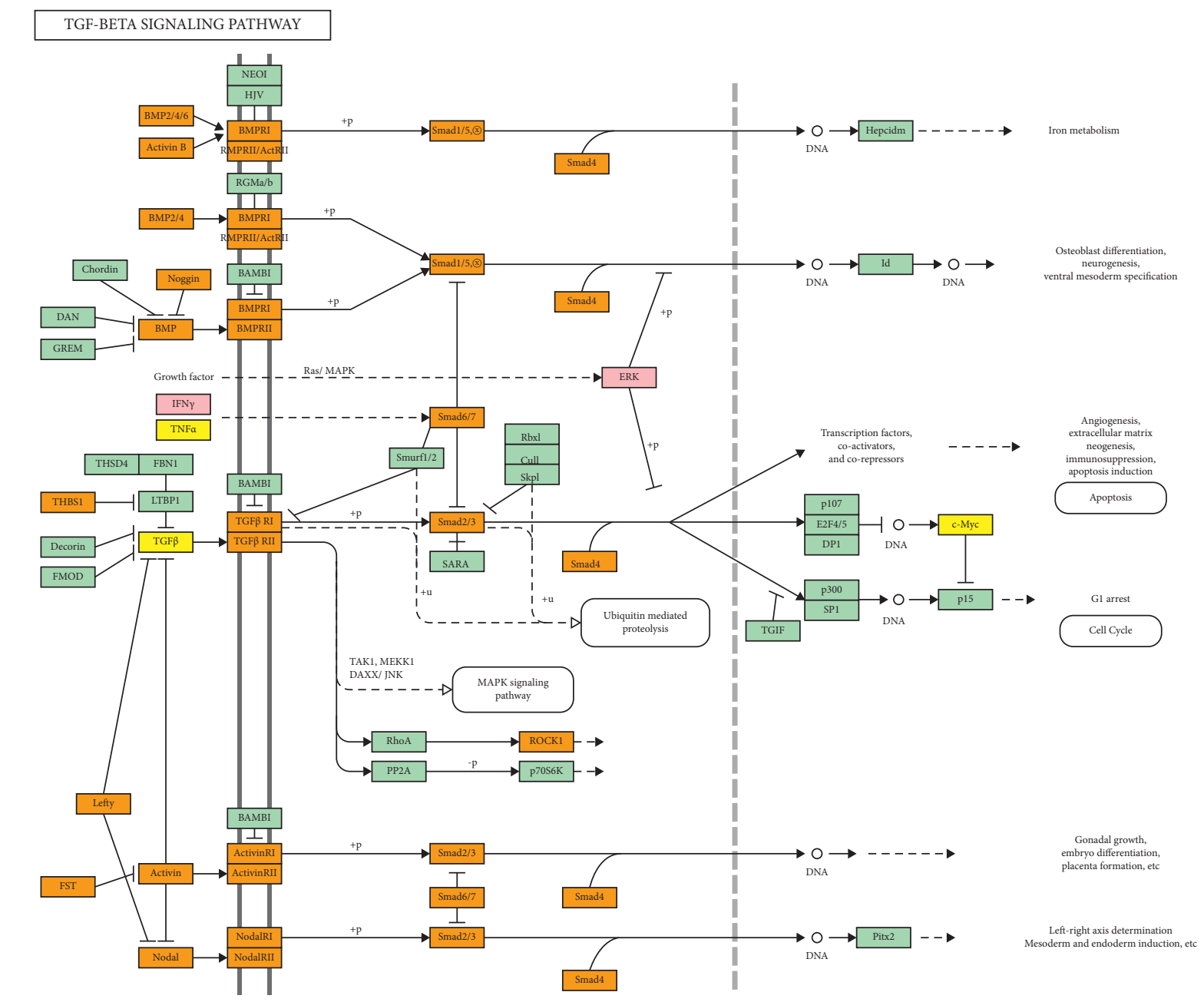

$043508 / 11 / 20$

(c) Kanehisa Laboratories

FIgUre 8: The KEGG Mapper modified from hsa04350 (pink is MTHSWD target, yellow is POF genes, and orange is MTHSWD-POF target).
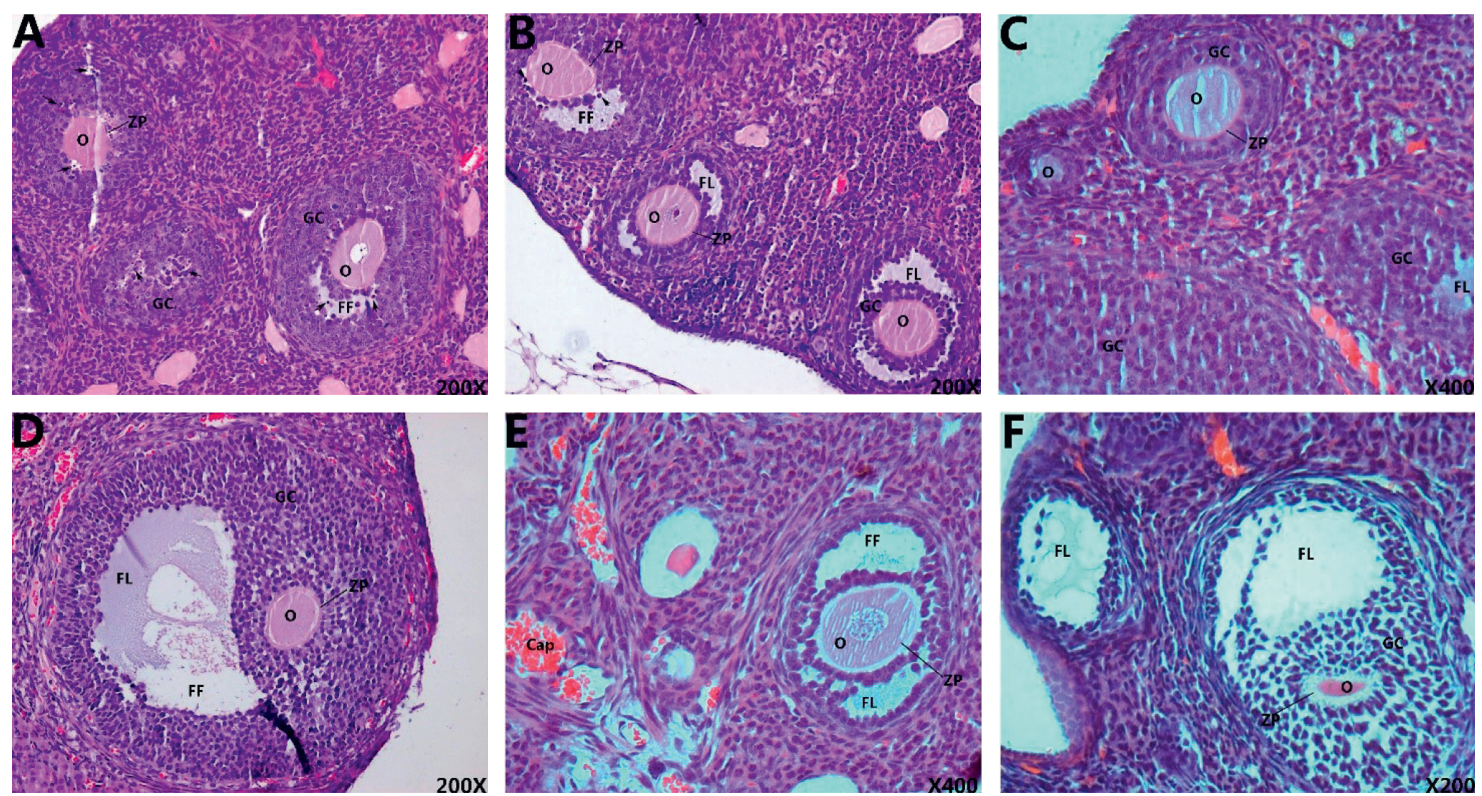

FIgURE 9: Morphological changes of ovarian tissue (HE staining: 200X). (a) Model group, (b) MTHSWD low-dose group, (c) MTHSWD medium-dose group, (d) MTHSWD high-dose group, (e) positive control group, and (f) blank group. O: oocyte, FL: follicular fluid, FF: follicular cavity, GC: granular cell, ZP: zona pellucida, Cap: blood vessel, and black arrow points to white blood cell. 


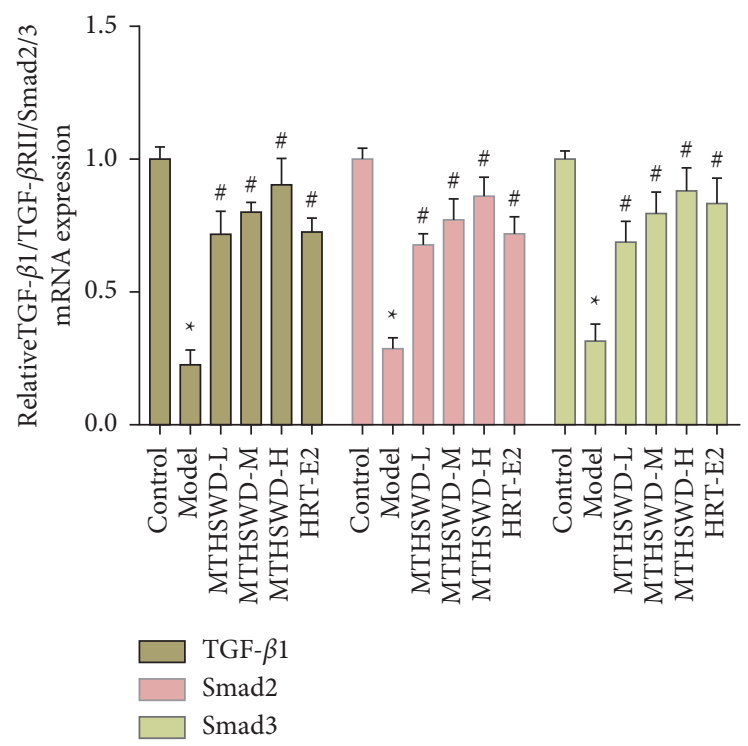

FIgURE 10: Expression of Smad2, Smad3, and Smad7 mRNA in ovarian tissue (compared with normal group, ${ }^{*} p<0.05$. Compared with model group, $\left.{ }^{\#} P<0.05\right)$.
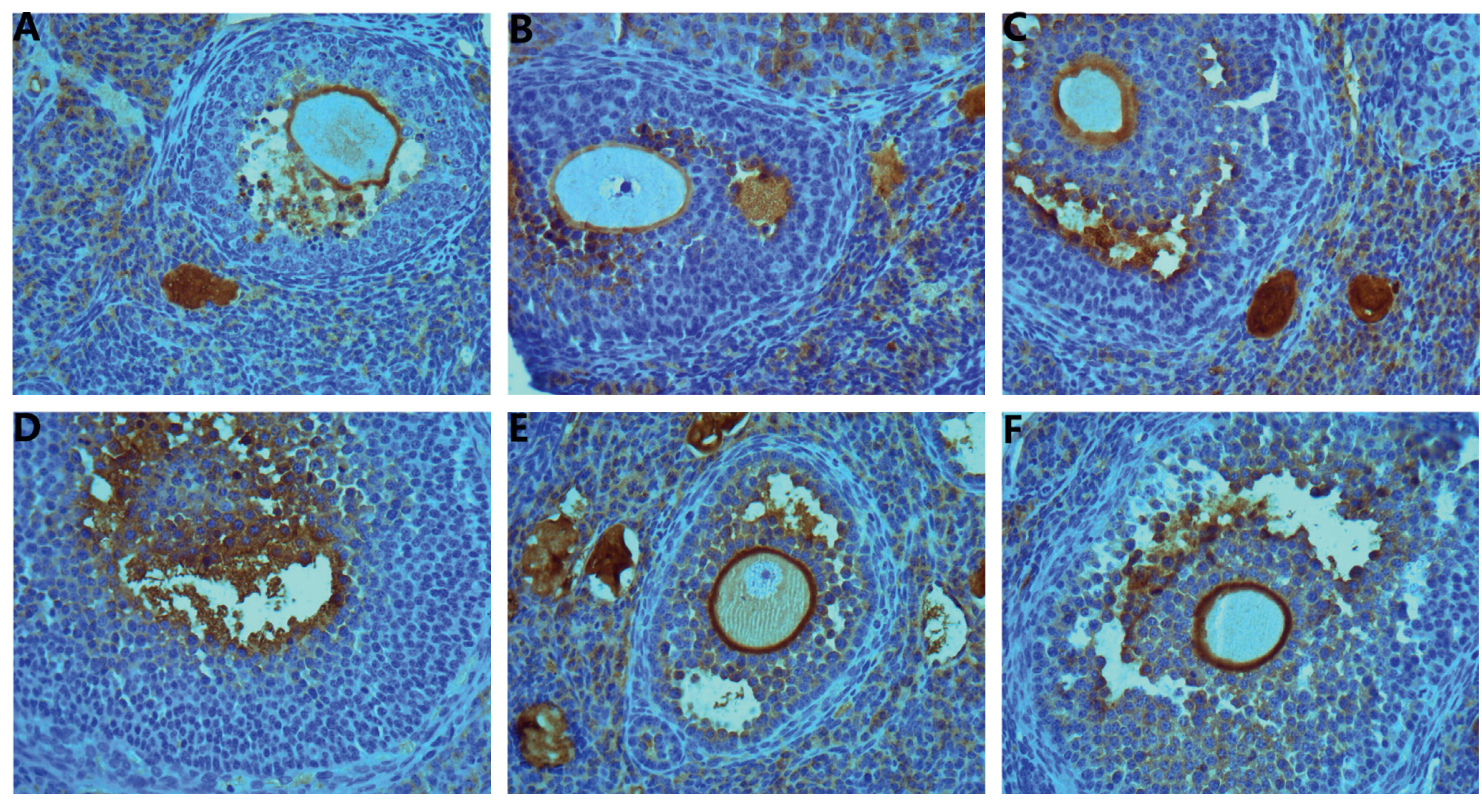

FIgURE 11: Expression of TGF- $\beta 1$ in granulosa cells (immunohistochemistry: 400X). (a) Model group, (b) MTHSWD low-dose group, (c) MTHSWD medium-dose group, (d) MTHSWD high-dose group, (e) positive control group, and (f) blank group.

group was significantly reduced, and the follicular atresia was significantly increased. After MTHSWD intervention, the protein expression of TGF- $\beta 1$, TGF- $\beta$ RII, and Smad $2 / 3$ was significantly increased $(P<0.05)$, and the expression of MTHSWD high-dose group was significantly higher than that of MTHSWD low-dose group $(P<0.05)$. 

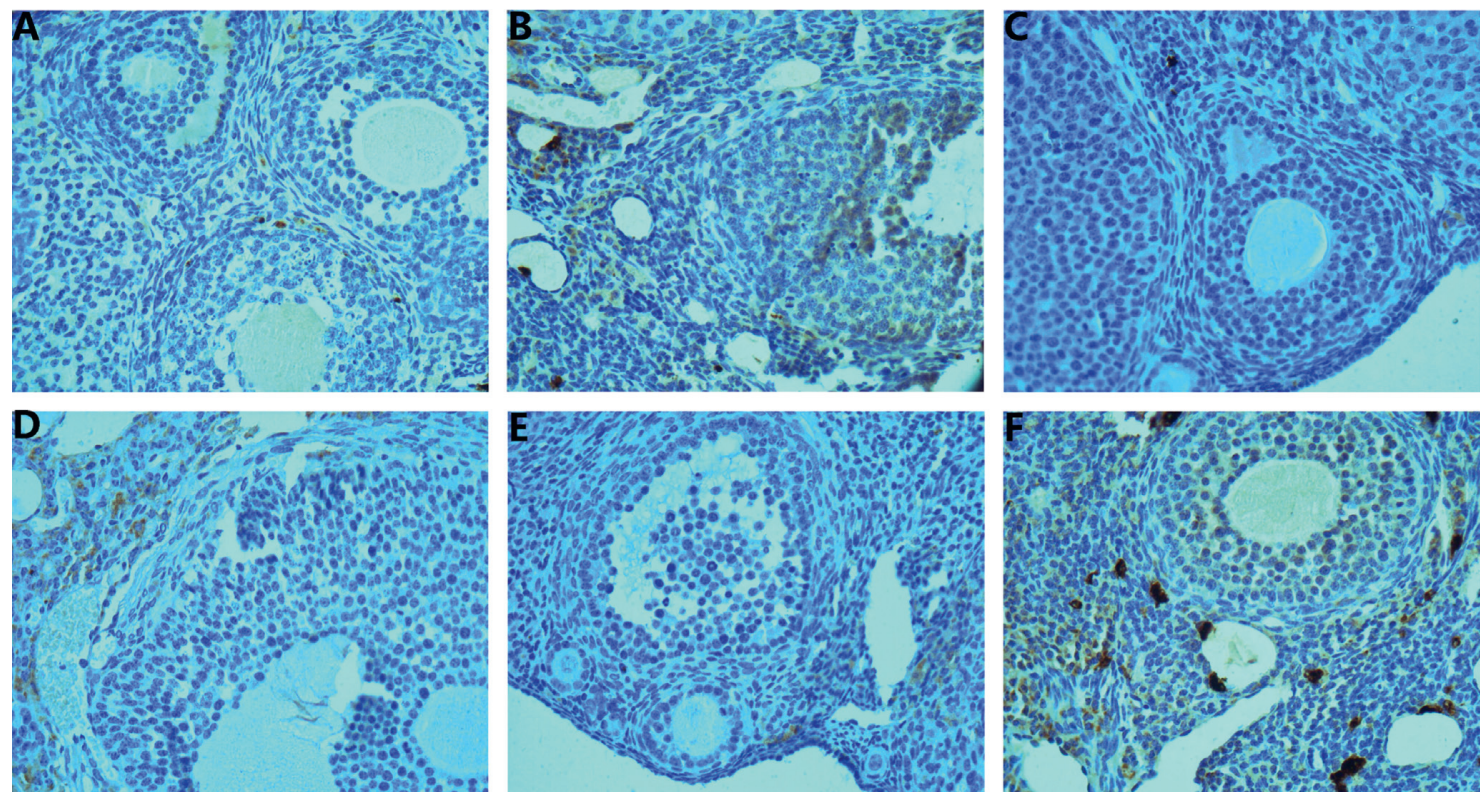

FIGURE 12: Expression of TGF- $\beta$ RII in granulosa cells (immunohistochemistry: 400X). (a) Model group, (b) MTHSWD low-dose group, (c) MTHSWD medium-dose group, (d) MTHSWD high-dose group, (e) positive control group, and (f) blank group.
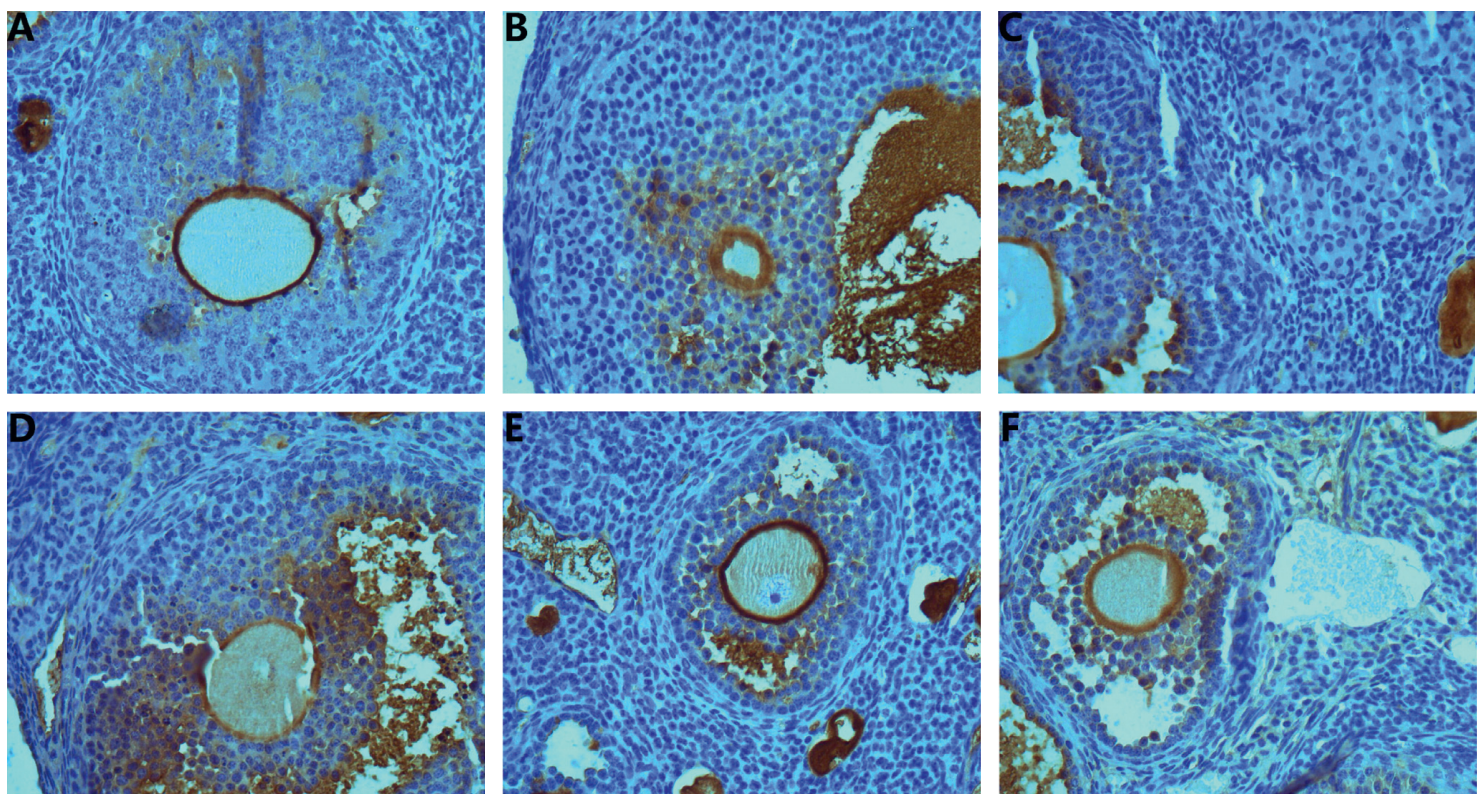

Figure 13: Expression of Smad2/3 in granulosa cells (immunohistochemistry: 400X). (a) Model group, (b) MTHSWD low-dose group, (c) MTHSWD medium-dose group, (d) MTHSWD high-dose group, (e) positive control group, and (f) blank group. 


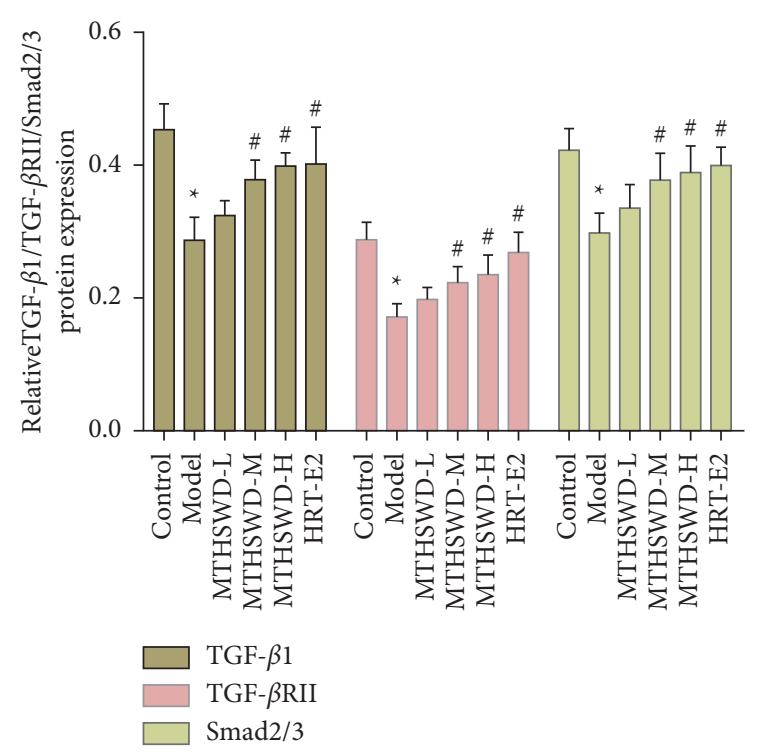

FIGURE 14: Expression of TGF- $\beta 1$, TGF- $\beta$ RII, and Smad $2 / 3$ proteins in granulosa cells (compared with normal group, ${ }^{*} p<0.05$. Compared with model group, ${ }^{*} P<0.05$ ).

\section{Conclusion}

In summary, this study showed that MTHSWD can significantly promote the transmission of the TGF- $\beta 1 / \mathrm{Smads}$ signaling pathway in POF mice, thereby promoting the proliferation and differentiation of granulosa cells. However, since we only studied the TGF- $\beta 1$ ligand in the TGF- $\beta$ pathway, the changes in the local microenvironment formed by the interaction of various factors within the ovary during the development of POF still need to be clearly elucidated. Meanwhile, whether MTHSWD can enhance the signal transcription of other cytokines by increasing the expression of Smads in the treatment of POF mice remains to be further studied.

\section{Data Availability}

All datasets for this study are included in the manuscript and the supplementary files.

\section{Disclosure}

Hunan University of Chinese Medicine is the first and corresponding address.

\section{Conflicts of Interest}

All authors have no financial or scientific conflicts of interest with regard to the research described in this manuscript.

\section{Authors' Contributions}

K. Yang, X. Yuan, W. Xiang, H. Liu, and G. Zhang participated in the concept and design. K. Yang, X. Yuan, Q. He, W. Xiang, and J. Fan are responsible for data analysis and interpretation in the network pharmacological section.
H. Liu and G. Zhang are responsible for data analysis and interpretation in experiments. K. Yang and Y. Xiao drafted the paper. G. Zhang and H. Liu supervised the study. All authors participated in the analysis and interpretation of data and approved the final paper.

\section{Acknowledgments}

This research was supported by Key Project of Scientific Research Fund of Hunan Provincial Education Department (nos. 19A370 and 21A0233), Hunan Province Domestic First-Class Cultivation Discipline Integrated Traditional Chinese and Western Medicine Discipline Project (no. 2021ZXYJH10), Key Projects of Hunan Province Traditional Chinese Medicine Scientific Research Program (no. 2021025), and Key Projects of the Scientific Research Fund of Hunan University of Chinese Medicine (no. 2020XJJJ003).

\section{Supplementary Materials}

Figure S1: the results of HPLC. Table S1: components and targets of MTHSWD. Table S2: POF genes. Table S3: enrichment analysis of MTHSWD-POF PPI network. (Supplementary Materials)

\section{References}

[1] S. Tsiligiannis, N. Panay, and J. C. Stevenson, "Premature ovarian insufficiency and long-term health consequences," Current Vascular Pharmacology, vol. 17, no. 6, pp. 604-609, 2019.

[2] V. Wesevich, A. N. Kellen, and L. Pal, "Recent advances in understanding primary ovarian insufficiency," F1000Research, vol. 9, p. 1101, 2020.

[3] S. Jaillard, K. Bell, L. Akloul et al., "New insights into the genetic basis of premature ovarian insufficiency: novel causative variants and candidate genes revealed by genomic sequencing," Maturitas, vol. 141, pp. 9-19, 2020.

[4] J. Paciuc, "Hormone therapy in menopause," Advances in Experimental Medicine \& Biology, vol. 1242, pp. 89-120, 2020.

[5] F. L. Gersh and C. J. Lavie, "Details on hormone replacement therapy," Heart, vol. 106, no. 16, pp. 2-1279, 2020.

[6] S. Azam, K. K. Jacobsen, A. R. Aro, E. Lynge, and Z. J. Andersen, "Hormone replacement therapy and mammographic density: a systematic literature review," Breast Cancer Research and Treatment, vol. 182, no. 3, pp. 555-579, 2020.

[7] R. Pirhadi, V. Sinai Talaulikar, J. Onwude, and I. Manyonda, "It is all in the name: the importance of correct terminology in hormone replacement therapy," Post Reproductive Health, vol. 26, no. 3, pp. 142-146, 2020.

[8] L. Cai, D. K. Zong, G. Q. Tong, and L. Li, "Apoptotic mechanism of premature ovarian failure and rescue effect of Traditional Chinese Medicine: a review," Journal of traditional Chinese medicine $=$ Chung $i$ tsa chih ying wen pan, vol. 41, no. 3, pp. 492-498, 2021.

[9] J. Lin, X.-l. Li, H. Song et al., "A general description for Chinese medicine in treating premature ovarian failure," Chinese Journal of Integrative Medicine, vol. 23, no. 2, pp. 91-97, 2017. 
[10] J. Lin, D. Wu, L. Jia et al., "The treatment of complementary and alternative medicine on premature ovarian failure," $E v$ idence-based Complementary and Alternative Medicine, vol. 2021, pp. 1-14, 2021.

[11] Y. J. Shang, Y. Y. Chen, and Su Lu, "Systematic evaluation and Meta-analysis of Chinese herbal medicine for the treatment of premature ovarian failure by tonifying the kidney and activating blood," Journal of Traditional Chinese Medicine, vol. 59, no. 15, pp. 1295-1299, 2018, in chinese.

[12] X. Y. Li, Y. J. Cao, P. Yang, and T. S. Su, "Meta-analysis of the efficacy of needle-drug combination in the treatment of earlyonset ovarian insufficiency," World Chinese Medicine, vol. 16, no. 13, pp. 1982-1989+1997, 2021, in chinese.

[13] L. L. Su, Clinical Observation on the Treatment of Early-Onset Ovarian Insufficiency with Kidney Deficiency and Liver Depression by Guiyuan Yijing Tang, Fujian University of Traditional Chinese Medicine, Fuzhou, China, 2021, in chinese.

[14] Y. B. Xu, M. M. Li, H. Y. An et al., "Meta-analysis of acupuncture combined with traditional Chinese medicine in the treatment of premature ovarian failure," Modern Chinese medicine clinical, vol. 27 , no. 05 , pp. $49-54+67,2020$, in chinese.

[15] M. D. Xie, X. F. Guo, and J. Tang, "Principles and research progress of Chinese medicine in the treatment of premature ovarian failure," Guangming TCM, vol. 36, no. 13, pp. 2271-2273, 2021, in chinese.

[16] L. Gao, Meta-analysis and Clinical Observation of Chinese Medicine Combined with Hormone Supplementation in the Treatment of Early-Onset Ovarian Insufficiency, Shandong University of Traditional Chinese Medicine, Jinan, China, 2018, in chinese.

[17] L. Liu, J. A. Duan, S. L. Su, P. Liu, Y. P. Tang, and D. W. Qian, "[Siwu series decoctions for treating primary dysmenorrea of gynecology blood stasis syndrome--research progress of TaohongSiwu decoction]," Zhongguo Zhongyao Zazhi, vol. 40, no. 5, pp. 814-821, 2015.

[18] J. Li, J. F. Shi, X. Nie, H. J. Jiang, C. M. Fu, and Y. He, "[Network pharmacology-based study on mechanism of Siwu Decoction in treatment of primary dysmenorrhea]," Zhongguo Zhongyao Zazhi, vol. 44, no. 3, pp. 559-565, 2019, in chinese.

[19] H. R. Ji, K. S. Park, H. L. Woo et al., "Herbal medicine (Taohong Siwu Tang) for the treatment of primary dysmenorrhea: a systematic review and meta-analysis," Explore, vol. 16, no. 5, pp. 297-303, 2020.

[20] P. Wu, "Observation on the efficacy of Siwu Wu Zi Tang combined with Western medicine artificial cycle for premature ovarian failure Zhejiang," Journal of Integrative Medicine, vol. 28 , no. 1 , pp. 34-37, 2018, in chinese.

[21] R. Xu, "Clinical observation on the treatment of premature ovarian failure with Wu Zi Yan Zong Wan combined with Si Wu Tang plus flavor," Combined Chinese and Western Medicine Research, vol. 11, no. 1, pp. 28-29, 2019, in chinese.

[22] M. J. Qiu and X. M. Xie, "Clinical study on the treatment of ovarian reserve hypofunction by adding Siwei Erxian Tang," Shaanxi TCM, vol. 42, no. 7, pp. 860-862, 2021, in chinese.

[23] Y. L. Deng, Y. Y. Huang, X. Q. Sang, L. Tian, and Z. J. Chen, "Effect of Shou Fetal Pill combined with Tao Hong Si Wu Tang on pregnancy quality and immune factors during treatment of infertility patients," Chinese herbal medicine, vol. 2021, no. 7, pp. 1764-1766, 2021, in chinese.

[24] G.-C. Huang, Y.-Z. Tsai, C.-J. Lee, H.-Y. Chang, and C.-C. Wang, "Elucidation of the effects of Si-Wu Tang on menstrual disorder patterns through activation of aromatase and antioxidation," Evidence-based Complementary and Alternative Medicine, vol. 2019, Article ID 4761651, 8 pages, 2019.

[25] C.-J. Lee, A. M. Kapelemera, Y.-Z. Tsai, C.-T. Lee, M.-Y. Xu, and C.-C. Wang, "Evaluating the therapeutic efficacy of SiWu-Tang decoction and concentrated extract in follicular maldevelopment-related menstrual disorders through pharmacokinetic/pharmacodynamic studies," Frontiers in Pharmacology, vol. 11, p. 1245, 2020.

[26] D. Zhao, Y. Yi, Q. He, S. Wang, K. Yang, and J. Ge, "Exploring the regulatory mechanism of Nao Tai Fang on vascular Dementia's biological network based on cheminformatics and transcriptomics strategy," Journal of Ethnopharmacology, vol. 274, p. 114065, 2021.

[27] K. Yang, L. Zeng, A. Ge et al., "Exploring the regulatory mechanism of hedysarum multijugum maxim.-chuanxiong rhizoma compound on HIF-vegf pathway and cerebral ischemia-reperfusion injury's biological network based on systematic pharmacology," Frontiers in Pharmacology, vol. 12, p. $601846,2021$.

[28] K. Yang, L. Zeng, A. Ge, Y. Yi, S. Wang, and J. Ge, "Exploring the oxidative stress mechanism of buyang huanwu decoction in intervention of vascular dementia based on systems biology strategy," Oxidative Medicine and Cellular Longevity, vol. 2021, Article ID 8879060, 29 pages, 2021.

[29] X. Yuan, Y. Wu, K. Yang, H. Liu, and G. Zhang, "Exploring the effect of jiawei buguzhi pills on TGF- $\beta$-smad pathway in postmenopausal osteoporosis based on integrated pharmacological strategy," Evidence-based Complementary and Alternative Medicine, vol. 2021, Article ID 5556653, 18 pages, 2021.

[30] J. Ru, P. Li, J. Wang et al., "TCMSP: a database of systems pharmacology for drug discovery from herbal medicines," Journal of Cheminformatics, vol. 6, no. 1, p. 13, 2014.

[31] J. S. Amberger, C. A. Bocchini, F. Schiettecatte, A. F. Scott, and A. Hamosh, "OMIM.org: online mendelian inheritance in man (OMIM), an online catalog of human genes and genetic disorders," Nucleic Acids Research, vol. 43, no. D1, pp. D789-D798, 2015.

[32] G. Stelzer, N. Rosen, I. Plaschkes et al., "The GeneCards suite: from gene data mining to disease genome sequence analyses," Current Protocols in Bioinformatics, vol. 54, no. 1, 2016.

[33] D. Szklarczyk, A. L. Gable, K. C. Nastou et al., "The STRING database in 2021: customizable protein-protein networks, and functional characterization of user-uploaded gene/measurement sets," Nucleic Acids Research, vol. 49, no. D1, pp. D605-D612, 2021.

[34] K. Yang, L. Zeng, A. Ge et al., "The effect of hedysarum multijugum maxim.-chuanxiong rhizoma compound on ischemic stroke: a research based on network and experimental pharmacology," Oxidative Medicine and Cellular Longevity, vol. 2020, Article ID 6072380, 24 pages, 2020.

[35] K. Yang, L. Zeng, A. Ge et al., "Systems biology and chemoinformatics-based strategies to explore the biological mechanism of fugui wenyang decoction in treating vascular dementia rats," Oxidative Medicine and Cellular Longevity, vol. 2021, Article ID 6693955, 42 pages, 2021.

[36] A. Al Shoyaib, S. R. Archie, and V. T. Karamyan, "Intraperitoneal route of drug administration: should it be used in experimental animal studies?" Pharmaceutical Research, vol. 37, no. 1, p. 12, 2019.

[37] A. Nair and S. Jacob, "A simple practice guide for dose conversion between animals and human," Journal of Basic and Clinical Pharmacy, vol. 7, no. 2, pp. 27-31, 2016. 
[38] X. Luo, H. M. Chang, Y. Yi, P. C. K. Leung, and Y. Sun, "Bone morphogenetic protein 2 upregulates SERPINE2 expression through noncanonical SMAD2/3 and p38 MAPK signaling pathways in human granulosa-lutein cells," The FASEB Journal, vol. 35, no. 9, p. e21845, 2021.

[39] L. J. Zhang, S. Y. Tao, P. W. Zhao, and X. L. Cong, "Experimental study on the apoptosis pathway to explore Erxian Tang in the treatment of premature ovarian failure in rats," World Science and Technology-Modernization of Chinese Medicine, vol. 17, no. 04, pp. 812-818, 2015, in Chinese.

[40] H. Cao, Z. L. Li, and M. Yao, "Effects of a herbal compound to benefit Qi and tonify blood on ovarian TGF- $\beta$ superfamily signaling pathway in mice with primary ovarian insufficiency," Chinese herbal medicine, vol. 25, no. 7, pp. 1-6, 2021, in chinese.

[41] L. Fang, Study on the Regulatory Effects of EGF-Related Factors and TGF-B1 on Ovulation-Related Gene Expression and Related Signaling Pathways in Human Granulosa Cells, Zhengzhou University, Zhengzhou, China, 2015, in chinese.

[42] X. N. Wu, L. E. Chen, Y. E. Wang, Y. Liu, and J. Qiao, “The mechanism of TGF- $\beta /$ Smads signaling pathway in gynecological diseases and the research progress of Chinese medicine intervention," Journal of Traditional Chinese Medicine, vol. 49, no. 1 , pp. 89-93, 2021, in chinese.

[43] M. Abdel Mouti and S. Pauklin, "TGFB1/INHBA homodimer/nodal-SMAD2/3 signaling network: a pivotal molecular target in pdac treatment," Molecular Therapy, vol. 29, no. 3, pp. 920-936, 2021.

[44] C. Liu, H.-M. Chang, Y. Yi et al., "ALK4-SMAD2/3-SMAD4 signaling mediates the activin A-induced suppression of PTX3 in human granulosa-lutein cells," Molecular and Cellular Endocrinology, vol. 493, p. 110485, 2019.

[45] H. Li, H.-M. Chang, Z. Shi, and P. C. K. Leung, "SNAIL mediates TGF- $\beta 1$-induced downregulation of pentraxin 3 expression in human granulosa cells," Endocrinology, vol. 159, no. 4, pp. 1644-1657, 2018.

[46] M. Kriseman, D. Monsivais, J. Agno, R. P. Masand, C. J. Creighton, and M. M. Matzuk, "Uterine double-conditional inactivation ofSmad2andSmad3in mice causes endometrial dysregulation, infertility, and uterine cancer," Proceedings of the National Academy of Sciences, vol. 116, no. 9, pp. 3873-3882, 20196.

[47] F. Tepekoy and G. Akkoyunlu, "The interaction of Wnt signaling members with growth factors in cultured granulosa cells," Animal Reproduction, vol. 17, no. 2, p. e20190106, 2020.

[48] J. A. Le Good, K. Joubin, A. J. Giraldez et al., "Nodal stability determines signaling range," Current Biology, vol. 15, no. 1, pp. 31-36, 2005.

[49] D. M. Leerberg, R. E. Hopton, and B. W. Draper, "Fibroblast growth factor receptors function redundantly during zebrafish embryonic development," Genetics, vol. 212, no. 4, pp. 1301-1319, 2019.

[50] C. Yu, Y.-L. Zhang, and H.-Y. Fan, "Selective Smad4 knockout in ovarian preovulatory follicles results in multiple defects in ovulation," Molecular Endocrinology, vol. 27, no. 6, pp. 966-978, 2013.

[51] E. A. Adu-Gyamfi, Y.-B. Ding, and Y.-X. Wang, "Regulation of placentation by the transforming growth factor beta superfamily $\dagger \dagger, "$ Biology of Reproduction, vol. 102, no. 1, pp. 18-26, 2020.

[52] M. J. D’Occhio, G. Campanile, and P. S. Baruselli, “Transforming growth factor- $\beta$ superfamily and interferon- $\tau$ in ovarian function and embryo development in female cattle: review of biology and application," Reproduction, Fertility and Development, vol. 32, no. 6, pp. 539-552, 2020.

[53] Y.-L. Chu, Y.-R. Xu, W.-X. Yang, and Y. Sun, "The role of FSH and TGF- $\beta$ superfamily in follicle atresia," Aging, vol. 10, no. 3, pp. 305-321, 2018.

[54] J. Yin, H.-M. Chang, Y. Yi, Y. Yao, and P. C. K. Leung, "TGF$\beta 1$ increases GDNF production by upregulating the expression of GDNF and furin in human granulosa-lutein cells," Cells, vol. 9, no. 1, p. 185, 2020.

[55] X.-Y. Zhang, H.-M. Chang, H. Zhu, R.-Z. Liu, and P. C. K. Leung, "BMP6 increases TGF- $\beta 1$ production by upregulating furin expression in human granulosa-lutein cells," Cellular Signalling, vol. 55, pp. 109-118, 2019.

[56] L. C. Poulsen, J. A. Bøtkjær, O. Østrup et al., “Two waves of transcriptomic changes in periovulatory human granulosa cells," Human Reproduction, vol. 35, no. 5, pp. 1230-1245, 2020. 